\title{
Corela
}

Cognition, représentation, langage

HS-19 | 2016

Le point de vue pris au mot

\section{Mais que me chantez-vous là ? Abus de langue et abus de langage}

\section{Laetitia Pille}

\section{(2) OpenEdition}

\section{Journals}

\section{Édition électronique}

URL : http://journals.openedition.org/corela/4426

DOI : $10.4000 /$ corela.4426

ISSN : 1638-573X

\section{Éditeur}

Cercle linguistique du Centre et de I'Ouest - CerLICO

\section{Référence électronique}

Laetitia Pille, « Mais que me chantez-vous là? Abus de langue et abus de langage », Corela [En ligne], HS-19 | 2016, mis en ligne le 08 juin 2016, consulté le 30 avril 2019. URL : http:// journals.openedition.org/corela/4426 ; DOI : 10.4000/corela.4426

Ce document a été généré automatiquement le 30 avril 2019.

\section{(c) (i) (2)(2)}

Corela - cognition, représentation, langage est mis à disposition selon les termes de la licence Creative Commons Attribution - Pas d'Utilisation Commerciale - Partage dans les Mêmes Conditions 4.0 International. 


\title{
Mais que me chantez-vous là? Abus de langue et abus de langage
}

\author{
Laetitia Pille
}

\section{Introduction}

1 L'assimilation de la langue ou du langage à la musique est fréquente et présente certainement un grand nombre d'avantages pratiques, dont celui, en apparence du moins, de faciliter par l'analogie la compréhension du propos. C'est sans doute pour cette raison qu'on trouve fréquemment, et dès l'Antiquité, ce procédé. Dans le Philèbe, par exemple, une analogie met en regard musique et parole.

SOCRATE - Le son que nous émettons par la bouche est un chez tous et chacun de nous, et, d'autre part, est d'une diversité infinie.

PROTARQUE - Assurément.

SOCRATE - et ni l'une ni l'autre chose ne suffit encore à nous rendre savants, soit de le connaître comme infini, soit de le connaître comme un ; mais connaître quelle quantité il a et quelles différences, voilà ce qui fait de chacun de nous un grammairien.

PROTARQUE - C'est très vrai.

SOCRATE - D'autre part, ce qui nous fait musicien, c'est la même chose.

PROTARQUE - Comment?

SOCRATE - Dans cet art aussi et pour autant qu'il en relève, le son est un.

PROTARQUE - Sans conteste.

SOCRATE - Posons donc deux premiers tons, l'aigu et le grave, et, comme troisième, le ton intermédiaire. Admets-tu cela?

PROTARQUE - Oui.

SOCRATE - Eh bien, savoir simplement cela ne serait pas encore connaître la musique, alors que l'ignorer ferait de toi, pour ainsi dire, une nullité dans cet art. PROTARQUE - En effet.

SOCRATE - Quant au contraire, mon ami, tu auras saisi quel nombre précis d'intervalles il y a dans le son relativement à l'aigu et au grave, quels sont ces intervalles, quelles sont leurs limites, combien de combinaisons en résultent, que 
les anciens ont reconnues et nous ont transmises, à nous leurs successeurs, avec leur nom d'harmonies, quels autres rapports de ce genre se manifestent dans les mouvements du corps, rapports qui se mesurent par des nombres et qu'il faut, disent encore les anciens, appeler rythmes et mètres et nous devons ajoutent-ils, concevoir que pareil procédé s'impose pour l'étude de toute unité et multiplicité ; quand donc tu auras saisi cela, alors tu seras devenu savant, et quand tu auras maîtrisé, en l'étudiant de cette façon, quelque autre unité en n'importe quel domaine, alors, en ce domaine, tu seras devenu compétent; mais en rester à la multitude infinie des choses individuelles et à l'infinie multiplicité que chacune d'elle renferme, t'empêche, en chaque cas, d'en prendre une notion achevée, et de devenir un homme qui compte et qui fait nombre, vu que, pour aucune d'elles, tu n'as pu atteindre aucun nombre défini. (Platon $1978: 17)^{1}$

2 Nous voyons dans ce passage quelle direction prend Socrate pour circonscrire la connaissance en matière de musique. Il traite bien de musique, et non pas de phénomène sonore, puisque le début du passage évoque, lui, le son; Socrate passe ensuite à la musique et évoque les conditions nécessaires à sa connaissance. Pour prétendre maîtriser la musique, il convient d'en connaître à tout le moins ce que Socrate énumère : les rapports, les nombres, les intervalles... en somme ce qui est mesurable.

Platon, dans le Philèbe, par le truchement de Socrate, continue de faire vivre la tradition pythagoricienne qui veut voir le monde régi par la beauté des lois mathématiques : par conséquent, la musique - et en particulier la mise en valeur des rapports harmoniques qu'elle permet - est elle aussi essentiellement une illustration sonore des lois mathématiques. C'est une des pistes métaphoriques employées pour décrire la musique ; elle perdure encore. En effet, nous rencontrons fréquemment des rapprochements mathématiques / musique, comme langage / musique.

4 Si la musique n'est que rarement comparée à la peinture, c'est peut-être parce qu'on ne perçoit pas, dans l'art pictural, cette fonction communicationnelle que l'on croit déceler dans la musique comme dans les langues ou dans les langages. En revanche, si l'on remarque souvent des comparaisons et raccourcis entre musique et langues / langages, c'est peut-être à cause de ce point commun que la musique comme les langues ou les langages se feraient percevoir par les oreilles et qu'ils seraient appréhendés comme des moyens de transmettre un message.

5 Avant de vouloir montrer que la musique n'est pas un langage ${ }^{2}$, il semble essentiel de dissiper un malentendu, qui conduit à confondre, en premier lieu, langage et langue. Nous montrerons ensuite comment la musique pourrait être définie par discrimination et non par assimilation ; enfin, qu'elle est utilisée à des fins idéologiques visant à mettre au jour des universaux cognitifs.

\section{La langue n'est pas le langage}

6 L'ouvrage de grand public, une référence et un manuel «usuel » comme l'on peut considérer la Grammaire Méthodique du Français, par Martin Riegel, Jean-Christophe Pellat et René Rioul, dès les premiers paragraphes, fait état de la nécessité de distinguer, d'une part les langues et d'autre part, le langage. Cette distinction s'avère même indispensable pour comprendre la suite de leur ouvrage comme ce qu'ils disent au sujet des langues elles-mêmes.

Les langues sont des moyens de communication intersubjectifs et ce que l'on appelle le langage n'est autre que la faculté, proprement humaine et liée à des 
aptitudes cognitives biologiquement déterminées, d'apprendre et d'utiliser les systèmes symboliques que sont les langues. L'usage actuel des deux termes, notamment sous l'influence de l'anglais (qui ne dispose que du seul terme language), est si flottant qu'on ne peut leur assigner que des définitions justifiées par des choix théoriques. L'option proprement linguistique en la matière a été clairement formulée par E. Benveniste (1966, p. 19): «le langage, faculté humaine, caractéristique universelle et immuable de l'homme, est autre chose que les langues toujours particulières et variables, en lesquelles il se réalise. C'est des langues que s'occupe le linguiste, et la linguistique est d'abord la théorie des langues. Mais [...] les problèmes infiniment divers des langues ont ceci de commun qu'à un certain degré de généralité ils mettent toujours en question le langage ». (Riegel, Pellat et Rioul $2001: 1$ )

7 L'introduction de cette grammaire, qui considère la langue comme un outil de communication, à juste titre, poursuit en exposant - et en approuvant - le modèle communicationnel élaboré par Jakobson.

Or ce modèle confond cette fois et en partie les langues et les langages. Il a pourtant subi des critiques en nombre et de qualité suffisamment remarquable pour que l'on se passe de leur retranscription in extenso. Pour notre discussion, nous reprendrons des éléments de la critique de Rastier (1995) et de celle de Raccah (1998, 2004), éléments susceptibles d'alimenter directement notre démonstration qui visera par la suite à :

- distinguer les langues des langages;

- les distinguer autant que possible du langage - dont il vient d'être question et qui correspond à la faculté d'utiliser les langues ;

- distinguer les langues, le langage et les langages de la musique.

\subsection{Le sens n'est pas l'information : les objections de Rastier}

Parmi les objections de Rastier (1995) à l'encontre du modèle de Jakobson, nous retiendrons qu'il s'oppose à l'idée d'assimiler le sens à l'information. Comprenons que le sens concerne la langue tandis que l'information concerne le langage. La différence essentielle entre les deux repose sur l'absence de code dans les langues et la présence de code pour l'élaboration et la compréhension du langage; ainsi dans ce dernier cas pouvons-nous parler d'encodage et de décodage. C'est donc ce concept de code qui soustend une autre objection de Rastier: compte tenu de la complexité sémiotique des langues, le concept de code «ne peut concerner que les unités de première articulation (cf. le code Morse)» (Rastier 1995 : 157), et d'ailleurs, comme il le remarque plus loin, un message codé, une fois décodé, laisserait toujours une part à l'interprétation.

Rastier s'oppose également à la conception unilatérale du modèle de Jakobson: non seulement le récepteur joue aussi un rôle dans la création du message - il peut l'influencer - mais en outre, l'expérience fournit souvent l'occasion de le vérifier : «le message diffère pour l'émetteur et le récepteur » (Rastier 1995 : 161).

\section{2. Énoncés et situation : Les objections de Raccah}

11 Parmi les objections de Raccah au modèle jakobsonien de la communication, nous retiendrons celles qui lui permettent d'aboutir à la différenciation de langue et langage, non plus seulement au plan fonctionnel, mais plus radicalement au plan essentiel : les différences qui séparent langages formels et langues sont des différences d'essence et 
" obligent à considérer langues et langages comme deux genres différents, et non pas comme deux espèces du même genre » (Raccah 1998 : 242).

Chmelik réunit les trois différences principales relevées par Raccah :

1) le statut des énoncés,

2) la notion de vérité,

3) la variabilité de la signification d'une expression à l'intérieur d'une même phrase. (Chmelik $2007:$ 106)

Pour le statut des énoncés (1), nous devons comprendre que la signification d'une phrase $(a=b+c)$, en langage formel, n'est pas dépendante de la situation dans laquelle elle est produite. Le statut des énoncés n'est donc pas le même ; par conséquent, en langage, la distinction entre énoncé et phrase n'est plus pertinente.

La différence concernant la notion de vérité (2) s'avoisine de la critique de Rastier concernant le risque d'assimilation du sens à l'information. Un énoncé, en langue, n'a pas pour unique fonction de donner une information. De la même façon, la fausseté de l'énoncé formel « $\mathrm{a}=\mathrm{a}+\mathrm{b}$ » n'est pas assimilable au mensonge contenu dans l'énoncé «la lumière est éteinte ", alors même que la lumière est allumée. L'énoncé n'est pas faux, il édicte un mensonge.

15 La troisième différence est assez proche des précédentes; un élément du langage doit se comprendre indépendamment de sa place ; et indépendamment de sa place, il conserve la même valeur $(a+b=a x c)$. Les trois différences se comprennent bien ensemble, et s'alimentent les unes les autres. Pour l'illustrer, Raccah emprunte un exemple à Ducrot :

Il s'agit d'un vers d'Andromaque de Racine, dans lequel le roi Pyrrhus annonce à Andromaque qu'il ne l'attendra plus et qu'il a décidé de tuer Astyanax, le fils d'Andromaque, comme les grecs le lui demandent :

(6) Je meurs si je vous perds mais je meurs si j'attends

L'articulation avec mais ne permet pas que les deux occurrences de je meurs soient interprétées dans le même sens : s'il en était ainsi, elles ne pourraient évidemment pas avoir des orientations argumentatives opposées, comme le requiert la présence de mais. D'autre part, la présence de mais requiert aussi que l'orientation de la deuxième occurrence de je meurs soit présentée comme plus importante pour son locuteur. Il en résulte que Pyrrhus, en prononçant (6), présente la « seconde mort » comme plus importante, plus terrible que la première: c'est pour l'éviter (lui préférant la première) qu'il décide de tuer Astyanax. (Raccah 1998 : 244)

Dans cet exemple, nous voyons une même phrase "je meurs ", réalisée en deux énoncés différents. Si nous nous attachons au contenu strictement informationnel, le sens nous échappe ; et enfin, les deux énoncés n'ont pas le même sens, bien que réalisant la même phrase.

17 Pour revenir au schéma de la communication jakobsonien, ce que Raccah lui reproche, c'est justement d'assimiler langue et langage. Le schéma de la communication décrit le fonctionnement d'un langage : sens encodé, transmission du message codé, décodage du message. Or, en langue

(i) Il n'y a pas de sens avant l'énonciation

(ii) Il n'y a pas de codage

(iii) Il n'y a pas de transmission de message

(iv) Il n'y a pas de décodage

(v) Il n'y a pas même de possibilité de comparer les représentations mentales.

(Raccah 2005 : 213)

18 Nous venons de montrer, à travers la critique du modèle jakobsonien, en quoi diffèrent langue et langage. Le présent article et son propos ne requièrent pas davantage de 
définitions et de développement pour la compréhension de son propos; ainsi avons-nous rempli l'objectif de préciser simplement quelques éléments de terminologie qui permettront ensuite de mieux saisir nos arguments visant à dégager les idéologies sur lesquelles s'appuient certaines analogies.

Nous ne devrions pas avoir besoin d'aller plus loin pour montrer que la musique n'est ni une langue, ni même un langage.

\section{La musique n'est pas une langue}

Avant de distinguer musique et langage, nous distinguerons musique et langue. Nous pourrions essayer d'associer les caractéristiques de la langue, énumérées ci-dessus, à celles de la musique ; en effet, en musique,

- il n'a pas de sens avant la production,

- il n'y a pas de codage,

- pas de transmission de message,

- pas de décodage,

- et pas non plus de possibilité de comparer les représentations mentales.

21 Ce qui différencie la langue de la musique n'est donc pas à chercher de ce côté-là, mais plutôt du côté du sens et de la fonction essentielle de l'une et de l'autre.

\subsection{La communication}

La langue permet de communiquer du sens, la musique permet d'exprimer, peut-être, des émotions. Exprimer n'est pas synonyme de " communiquer ». Communiquer est «mettre en partage le sens d'un énoncé». Même si le sens du côté des récepteurs n'est pas toujours le même, d'un récepteur à l'autre, et n'est pas identique au sens voulu par l'émetteur (encore que, (1) il est bon de rappeler qu'il n'y a pas de sens en dehors de l'énoncé, donc pas de sens chez l'émetteur ou le récepteur, mais peut-être une interprétation différente, et mouvante. (2) nous ne pouvons émettre à ce sujet que des hypothèses puisque l'on ne peut observer, ni le sens, ni l'interprétation!) du moins, nous savons que les énoncés qui ont pour objectifs de témoigner au sujet de ce que l'on a ressenti à l'écoute d'une musique, ces énoncés ne sont pas identiques. La musique permet donc au musicien d'exprimer quelque chose, sans doute de l'ordre de l'émotion, mais elle ne lui permet pas de communiquer cette émotion. Pourrions-nous d'ailleurs vérifier une telle chose ? Sachant que notre émotion, à l'écoute d'une musique, varie peut-être autant de fois que nous écoutons cette musique. Plutôt que communiquer, la musique semblerait produire ou créer une émotion, un état.

\subsection{Les parties et le rôle de la répétition des parties}

À ces arguments, l'on pourrait opposer que, dans le cas des énoncés en langue, nous pouvons proposer quelques analogies: la structure des énoncés et leur répétition est aussi importante que la structure d'une "phrase » musicale ou de sa répétition pour la compréhension ou l'interprétation de l'ensemble. Un même énoncé ne sera pas non plus compris de la même façon par deux individus différents. Reprenons l'exemple de Racine cité plus haut : «Je meurs si je vous perds mais je meurs si j'attends ». 


\section{La musique n'est pas un langage}

24 entendons l'idée selon laquelle, dans les deux domaines, l'objectif est de communiquer quelque chose, un message, une émotion. La musique communique une émotion; grâce au langage (entendu ici comme faculté humaines d'utiliser les langues), nous pouvons également communiquer une émotion. Mais, non seulement nous pourrions faire l'objection que pour qu'il y ait communication, il faut qu'il y ait possibilité de retour ; or, lorsque l'on écoute une interprétation, on ne peut rien communiquer en réponse - sauf des applaudissements; mais en allant plus avant dans une description du langage, nous pourrions également nous demander si la fonction de communiquer une émotion pour le langage n'est pas un peu réductrice lorsqu'elle est ainsi choisie, parmi toutes les fonctions des langues, comme celle décisive pour justifier son assimilation à la musique.

À ce sujet, Popper dans La connaissance objective défend justement que les fonctions principales du langage ${ }^{3}$ ne sont pas la communication, mais plutôt la description et l'argumentation.

Les fonctions ou dimensions les plus importantes du langage humain (que les langages animaux ne possèdent pas) sont les fonctions descriptives et argumentatives. (Popper 1972 : 201)

La fonction argumentative du langage présuppose la fonction descriptive: les arguments portent, fondamentalement, sur des descriptions - ils critiquent les descriptions du point de vue des idées régulatrices de vérité, de contenu et de vérisimilitude.

Or deux points sont ici d'une importance capitale :

sans le développement d'un langage descriptif exosomatique, d'un langage qui, comme un outil, se développe à l'extérieur du corps - , il ne saurait exister aucun objet pour notre discussion critique. Mais, avec le développement d'un langage descriptif (et, plus tard, d'un langage écrit), un troisième monde linguistique peut émerger; et c'est uniquement par ce moyen, et uniquement dans ce troisième monde, que les problèmes et les normes de la critique rationnelle peuvent se développer.

C'est à ce développement des fonctions supérieures du langage que nous devons notre humanité, notre raison. Car nos pouvoirs de raisonner ne sont rien d'autre que les pouvoirs de l'argumentation critique.

Ce second point montre la futilité de toutes les théories du langage humain qui se concentrent sur l'expression et la communication. Comme nous le verrons, l'organisme humain, qui, on le dit souvent, est fait pour s'exprimer lui-même, dépend très largement dans sa structure de l'émergence des deux fonctions supérieures du langage. (Popper $1972: 200$ )

Popper est sans doute audacieux et on pourrait le trouver radical dans son jugement. Néanmoins, il nous semble à nous aussi judicieux de définir un phénomène en insistant justement sur ce qui le distingue d'un autre phénomène et non pas en faisant son essence de ce qui pourrait l'assimiler à un autre phénomène. Si nous pensons que l'essentiel du langage réside dans ses fonctions expressives et communicatives, alors effectivement, nous pouvons être tentés, du moins lors d'un premier examen sans doute superficiel, d'assimiler langage et musique. Si nous pensons que l'essentiel du langage réside dans ce qu'il présente comme caractéristiques particulières, à savoir la description et l'argumentation, alors il n'y a plus de confusion possible. Pour ce qu'il avance, Popper distingue également le langage humain du langage des animaux. 
Les langages humains partagent avec les langages animaux les deux fonctions inférieures du langage : (1) l'expression de soi et (2) l'échange de signaux.

Tous les langages animaux et tous les phénomènes linguistiques ont en commun ces deux fonctions inférieures. Mais le langage humain a bien d'autres fonctions. Assez étrangement, les plus importantes de ces fonctions supérieures ont été négligées par la plupart des philosophes. L'explication de ce fait étrange, c'est que les deux fonctions inférieures sont toujours présentes quand les fonctions supérieures le sont, si bien qu'il est toujours possible d'« expliquer » n'importe quel phénomène linguistique en le rapportant aux fonctions inférieures, c'est-à-dire comme une expression ou une communication.

Les deux fonctions supérieures les plus importantes des langages humains sont : (3)

la fonction de description et (4) la fonction d'argumentation ${ }^{4}$. (Popper 1972 : 199)

Nous pouvons produire la même remarque concernant le langage humain et la musique : si certains chercheurs assimilent la musique à un langage, c'est par commodité langagière, justement, en négligeant ce qui est, d'après Popper, particulier au langage à l'exclusion de tout autre domaine, (« langage » toujours entendu comme faculté humaine d'utiliser des langues) à savoir la fonction de description et la fonction d'argumentation.

\subsection{Le découpage et la mesure}

Néanmoins, pour justifier la métaphore, on remarque souvent que la musique comme les langues, ou plus exactement une "phrase » musicale comme une "phrase» en langue constitue des ensembles que l'on peut l'un et l'autre découper en parties, dont on peut isoler des éléments constitutifs. Or nous pensons que la possibilité de découper un ensemble en parties n'est pas une caractéristique suffisamment pertinente pour qu'elle permette, si elle est partagée par deux objets d'étude (en l'occurrence la langue et la musique) d'assimiler les deux ensembles (objets d'étude) qui ont en commun cette caractéristique.

En effet, si l'on découpe un morceau de musique en parties, en " phrases » musicales ou en notes, et si l'on trouve deux " phrases " similaires, ou même identiques à l'intérieur d'un même morceau, nous ne pourrons pas dire pour autant qu'elles auront le même " sens ", le même rôle, la même portée. Produiront-elles le même effet ou bien la seconde produira justement un premier effet de répétition, renforcé (donc différent) à chaque répétition de cette même "phrase "? Que dire d'une structure rythmique qui se répète tout le long d'un morceau? Cette structure, répétée, ne saurait être réduite à une occurrence; nous voyons bien que c'est justement sa répétition qui parfois donne un cadre ou un intérêt, un support ou une structure à un morceau de musique. De même, un élément de langage, en code morse par exemple, répété à l'identique plusieurs fois, n'aura pas d'autres fonctions que celle d'être répétée dans le but d'être mieux entendue. L'information qu'elle exprimera ne sera nullement modifiée ou amplifiée par sa répétition. (D'ailleurs, comment pourrait-on amplifier ou intensifier une information?) Pour ce qui regarde la langue et les énoncés en langue, l'argument est sensiblement différent, mais nous y reviendrons plus loin.

\subsection{Le message et le code...}

Est-ce que la musique transmet un message codé, qu'il nous serait possible de décoder, à l'aide d'un décodeur, de la clé ? Nous avons beau dire qu'une musique mineure sera plus triste qu'une majeure, sans parler des réalités auxquelles une musique pourrait nous 
renvoyer, chaque musique fait naître un univers spirituel ou mental différent, un état différent, fort dépendant du sujet auditeur. Ce que l'on imagine ou que l'on ressent n'est comparable ni d'une musique à l'autre, ni, pour une même musique, d'un auditeur à l'autre, ni même, parfois, pour une même musique et un même auditeur, mais une époque ou un moment et un lieu différents, d'un ressenti à l'autre.

Le passage que vous aimez particulièrement dans un morceau ne sera pas transposable dans un autre : transposé dans un autre, peut-être ne suscitera-t-il plus la même émotion.

\section{Le « langage musical » et les universaux cognitifs}

Si vraiment l'on voulait rapprocher la musique de l'un des deux, langue ou langage, ce n'est vraiment pas le langage qu'il faudrait évoquer. Or, curieusement, c'est justement « la musique est un langage » ou « le langage de la musique » que l'on entend ou que l'on lit le plus souvent.

33 Nous avons cherché à comprendre d'où provenait cette métaphore et si elle servait une idéologie.

\subsection{Le piège de la structure et de la construction}

Regardons de plus près quels sont les arguments en faveur de cet amalgame. Examinons l'introduction de Coeurdevey :

Faire œuvre de composition musicale, n'était-ce pas nécessairement se soumettre à un ensemble de règles, et celles-ci ne découlaient-elles pas de lois permettant de produire un moyen de communication, un langage précisément, avec son vocabulaire et sa syntaxe - vocabulaire et syntaxe certes singulièrement malmenés depuis un certain temps, mais dont personne ne s'était imaginé que l'on pût saper les fondements? Car, de même que toute langue ne cesse d'évoluer (en témoignent les ajouts de nos dictionnaires, admettant des mots nouvellement créés et des tournures jusque-là prohibées, sans que la nature même de cette langue soit pour autant remise en question), de même les compositeurs de la fin du XIX $\mathrm{X}^{\mathrm{e}}$ siècle - et beaucoup encore, même de nos jours - ont pu se livrer à toutes sortes d'explorations, jusqu'aux extrêmes confins du système, usant avec une liberté croissante de procédures irrégulières ou exceptionnelles, sans que, pour l'essentiel, les fondements du système en aient été ébranlés. (Coeurdevey 1998 :3)

L'abus de la comparaison apparaît clairement: l'auteur parle de langage musical parce qu'elle considère la musique comme un moyen de communication (et non une fin en soi), et parce qu'elle évoque le vocabulaire et la syntaxe de la musique, ce qui en réalité correspond aux unités de sons et à la façon dont, suivant les courants, lieux, époques, il est bon - ou mauvais - de les agencer. En fait, le point commun consiste dans le fait que, dans les deux cas, l'ensemble est constitué de parties agencées suivant des règles de construction propres à l'ensemble, pour que l'ensemble remplisse sa fonction. Autrement dit, l'on pourrait aussi bien, à ce compte-là, parler du langage de la tapisserie, du meuble, d'un moteur de voiture... c'est pourquoi nous ne croyons pas pertinent ce critère dans la comparaison musique / langage, bien que nous admettions que l'analogie présente l'avantage d'être fonctionnelle; il est facile et rapidement compréhensible par un grand nombre de désigner des éléments constitutifs de la musique par vocabulaire, phrase, syntaxe... simplifier est d'ailleurs un des rôles de l'analogie, mais certes pas définir... 

et ne sont présentées comme des systèmes de lois d'agencement des éléments qu'a posteriori, rétrospectivement. Des législateurs en matière de morale publique, dès l'Antiquité, édictaient ce qui était acceptable et ce qui ne l'était pas en matière d'harmonie ; leur but était principalement d'empêcher l'émergence de nouveaux modes. Les règles mathématiques qui régissent la musique et que l'on invoque souvent pour expliquer, tout aussi rapidement et abusivement, que la musique est mathématique, sont celles qui dirigent l'accompagnement d'une mélodie.

\subsection{Pour la fonction de communication}

Admettons que ce qui conduit l'auteur à parler de langage de la musique plus aisément que du langage d'un moteur de voiture soit la fonction de communication.

Fubini se trouve en butte au même souci de différenciation et de rapprochement lorsqu'il essaie de déterminer la part d'histoire et la part de nature dans la musique.

Naturalité et historicité semblent donc s'affronter comme deux thèses antithétiques et irréductibles sur la nature de la musique. Et en effet, nulle médiation ne paraît possible, à moins que l'on adopte une vision dialectique. (Fubini 1998 : 18)

et c'est là qu'intervient la mention qui nous intéresse :

Adorno l'a effectivement tenté dans son fameux « fragment sur les rapports entre musique et langage ». Adorno y affirme que " la musique vise un langage dépourvu d'intentions [...]. Une musique vide de toute intention, réduite à un simple kaléidoscope acoustique. Mais si elle ne faisait, à l'inverse, que vouloir dire quelque chose, elle cesserait d'être musique, et tournerait faussement à la parole. $\aleph^{5}$ Selon Adorno, la musique vit donc, dans cette perpétuelle tension dialectique, à la limite de la signification, de la communication verbale, nous pourrions ajouter du langage comme fait historique et déterminé, sans jamais tendre à s'identifier avec lui ; et d'autre part elle tend, à l'opposé, à se présenter comme pur objet, comme pure architecture de sons, comme forme, nous pourrions ajouter comme fait naturel, mais sans jamais s'y réduire totalement. (Fubini $1998: 18$ )

Même si nous ne comprenons pas pourquoi il y a une "tension dialectique » de la musique entre la signification et l'objet pur, la forme, nous comprenons néanmoins que cet auteur admet que l'intention de la musique, si elle en a une, n'est pas de vouloir dire quelque chose. Nous pourrions dire aussi que, puisque la musique n'est ni un moyen de communication, ni une pure forme acoustique, comme fait naturel, elle n'est pas à michemin entre les deux, mais tout simplement, elle n'est aucun des deux.

Cette position est défendue plus radicalement par Schloezer :

Il y a incompatibilité, opposition profonde entre la musique, système fermé, qui est la chose même qu'elle signifie, et le langage, système ouvert, qui n'est que l'expression de ce qu'il signifie, qui renvoie à un sens qui lui est transcendant et dont il reçoit son unité.

Le problème ne peut être tranché que par l'assimilation complète de l'un des deux systèmes par l'autre.

L'œuvre vocale est une, cohérente, parce que la parole s'y trouve totalement assimilée par la musique.

Le sens des paroles chantées n'est plus le même que celui qu'avaient ces mêmes paroles avant leur mise en musique... mais celui que leur confère la phrase musicale. (Schloezer $1947: 268$ )

Si j'écoute une œuvre chantée dans une langue étrangère, 
Ma compréhension de l'œuvre s'effectuera dans des conditions plus favorables, je ne serai pas tenté en effet d'en extraire les paroles qui y sont dissoutes pour chercher à savoir ce qu'elles veulent dire. (Schloezer $1947: 273$ )

Ruwet, lui, ne reprend Schloezer que pour s'en écarter :

N'est-il d'ailleurs pas curieux de voir Boris de Schloezer - qui a tant fait pour une approche structurale du phénomène musical, qui a montré que le sens d'une musique est à chercher dans sa structure et non dans son origine ou ses effets psychologiques - abandonner en définitive ce point de vue quand il s'agit de rendre compte de la présence des paroles dans la musique.

Et puis, la perspective adoptée par Boris de Schloezer se heurte à un fait indubitable, qu'elle tend à rendre incompréhensible: c'est l'existence même, et l'ubiquité, de la musique vocale, son prestige universel - dans bien des cultures, la musique purement instrumentale ne joue-t-elle pas un rôle subalterne? (Ruwet 1972: 43)

44 Nous ne nous lancerons pas dans la défense ou la critique de la position de Ruwet, cela nous conduirait en des développements trop éloignés de notre propos de départ. Notons toutefois que ce que Ruwet croit repérer comme un paradoxe $(l .1 \& 2)$ révèle bien plutôt la confusion de son approche : il fait comme si l'étude de la structure de la musique et concomitamment de la structure du langage aboutissait immanquablement à assimiler l'un à l'autre. Bien au contraire, il est fort intéressant que celui qui oppose fondamentalement musique et langage, Schloezer, soit lui aussi un défenseur de l'approche structurale; cela montre que l'on peut s'intéresser aux structures des deux productions sans pour autant les confondre.

Remarquons également que prétendre que le langage est bien différent de la musique n'entraîne pas nécessairement que les paroles en musique n'ont aucune portée supplémentaire, du fait de la musique. Ce que Schloezer défendait, c'est que la beauté des paroles n'augmenterait en rien, en revanche, l'intérêt porté à la musique. Quant au rôle, subalterne ou principal, joué dans la société par la musique instrumentale ou la musique vocale, nous ne voyons pas en quoi il peut être un critère déterminant ou pertinent dans l'effort de distinction entre musique et langage.

\subsection{La tentation des universaux cognitifs}

46 Les sciences cognitives et les recherches de structures universelles de la pensée peuvent aussi, à certains égards, contribuer à la pérennité de cette confusion entre langage ou langue (l'un et l'autre étant bien souvent employés comme s'ils étaient équivalents) et musique.

Meyer, à ce propos, commence par se défendre de s'intéresser à des universaux musicaux :

Pour expliquer pourquoi des êtres humains pris dans un contexte historico-culturel pensent, réagissent et choisissent comme ils le font, il faut distinguer les aspects du comportement humain qui sont appris et variables de ceux qui sont innés et universels. Mais c'est une erreur, - même si elle est très répandue - de conceptualiser ce problème comme la recherche d'universaux « musicaux ». Il n'y en a pas. Il n'y a que des universaux acoustiques du monde physique et les universaux bio-psychologiques du monde humain. Et si les stimuli acoustiques affectent la perception, la connaissance, et partant la pratique de la musique, ce n'est qu'à travers l'action contraignante des universaux bio-psychologiques. (Meyer $1998: 26)$ 
propose ensuite une liste de cinq points de ces universaux bio-psychologiques : "Les contraintes neuro-cognitives, les paramètres syntaxiques et statistiques, la classification, les structures hiérarchiques et la redondance. » (Meyer 1998 : 26).

Pour le premier point, Meyer explique que bon nombre des caractéristiques de la musique sont conditionnées par les facultés limitées du cerveau humain; peu d'entre nous parviennent à discriminer deux sons à intervalle d'un quart de ton (et même, la plupart d'entre nous n'entendent guère de nuance en deçà d'un demi-ton). Par conséquent, un compositeur ne jouera pas des intervalles inférieurs au quart de ton, sauf cas exceptionnels où cela correspondrait justement à un objectif. Évidemment, les conditions de production en langue sont elles aussi dépendantes de nos facultés cognitives, ne seraitce que parce que : «notre vitesse de traitement des informations et réponses musculaire est semblablement limitée. » (Meyer 1998 : 27).

Nous pourrions en dire autant de n'importe laquelle de nos qualités sensorielles : en effet il ne nous est pas permis, non plus, de voir davantage et mieux que ce que nos capacités visuelles et cognitives nous permettent de traiter.

Pour ce qui regarde les paramètres syntaxiques et statistiques, voilà ce qu'en dit Meyer :

En raison des capacités innées de l'esprit humain, on peut diviser certains paramètres $\mathrm{du}$ son en stimuli perceptibles séparément et en relation proportionnelle pour les utiliser comme fondements de structurations auditives. Dans la plupart des musiques du monde, c'est le cas avec la hauteur et la durée, qui sont les composantes de base de la mélodie, du rythme, de la métrique et (dans la musique occidentale) de l'harmonie. Dans la mesure où les probabilités et les possibilités bien connues qui régissent la succession de ces paramètres peuvent servir de fondement à la syntaxe, j'ai appelé ces paramètres 'syntaxiques'. (Meyer 1998 : 27)

et Meyer de relever son emploi du mot syntaxique en note :

Ceci attire notre attention sur le fait qu'il y a des aspects du comportement humain, comme la syntaxe du langage et de la musique, qui sont bien des universels dans le champ de la bio-psychologie, mais dont l'actualisation culturelle est variable, bien que soumises à des règles. (Meyer 1998 : 28)

Or, même ainsi justifié, le seul fait d'employer « syntaxe » pour désigner l'ensemble des règles qui permettent d'ordonner les éléments qui composent la musique comme les éléments qui composent la langue facilite l'amalgame entre ce qui caractérise la langue et ce qui caractérise la musique. De la même façon, et pour reprendre notre exemple grossier du moteur de voiture, l'agencement des parties d'un moteur de voiture répond à diverses contraintes, mécaniques et physiques par exemple, que l'on pourrait appeler, tout aussi abusivement, « syntaxe » des éléments du moteur.

On pourrait reprocher aux chercheurs d'universaux d'abuser de la syntaxe quand on en lit la définition du Petit Robert :

SYNTAXE : 1. GRAMM Étude des relations entre les formes élémentaires du discours (mot, syntagmes). COUR Étude des règles qui président à l'ordre des mots et à la construction des phrases, dans une langue; ces règles. > grammaire. Respecter la syntaxe. Syntaxe fautive. Les mots "sont esclaves et libres, soumis à la discipline de la syntaxe " (Mme de Staël). Étude descriptive des relations existant entre les unités linguistiques (dans le discours) et des fonctions qui leur dont attachées. Syntaxte et morphologie. Syntaxe et syntagmatique. Ouvrage de syntaxe > Grammaire. La Syntaxe de Sandfeld, de G. et R. Le Bidois. 2. Relations qui existent entre les unités linguistiques, considérées abstraitement (dans la langue) ou concrètement (dans la parole, le discours) "phrases bien construites (...) dont la syntaxe accoutumée règle l'ordonnance ». 
(Caillois). Étudier la syntaxe d'un tour, d'une expression, d'une phrase > construction. 3. En Belgique, Première année du « secondaire supérieur " précédant la rhétorique et la poésie. 4. INFORM. Ensemble des règles qui régissent l'écriture des instructions d'un programme dans un langage donné.

(Le nouveau Petit Robert 1993 : 2456) semble apparaître entre le sujet et l'objet : même si nous passons sur l'emploi abusif, une fois encore, de «langage » en lieu et place de « langue ", l'auteur énumère et répertorie ce qu'elle aimerait assimiler à des universaux en musique : la gamme et l'agencement des notes en une gamme dont les intervalles diffèrent, l'organisation des notes : «En outre, l'organisation des notes dont les hauteurs montent et descendent dessine un contour mélodique, dont l'importance se retrouve dans les musiques de toutes les cultures. " (Tillman $2008:$ 125). caractéristiques importantes : le rythme, la mesure et le tempo.

À partir de ces constats, voici la question que se pose le chercheur :

Diverses structures musicales se retrouvent donc dans toutes les cultures. Pourquoi? Certains invariants résultent vraisemblablement de la façon dont le cerveau traite les sons. Quelles sont donc ces relations? Peut-on en déduire des invariants cognitifs? (Tillman $2008: 125$ )

En dehors du fait qu'il ne nous semble pas qu'il s'agisse ici de « déduction », mais plutôt d'induction, nous nous interrogeons sur la nature des "relations » entre musique et cerveau. C'est d'ailleurs ainsi que commence le paragraphe suivant :

La musique, comme n'importe quel son, est traitée par le cerveau qui possède certaines propriétés d'organisation, d'apprentissage, de mémorisation et d'attention. Voyons quelques-unes de ces caractéristiques en relation avec la façon dont est construite la musique. (Tillman $2008: 126)$

63 À cela, d'une part, nous pouvons objecter que la musique est sans doute traitée par la pensée plutôt que par le cerveau. D'autre part, il nous semble que, dans cette présentation du traitement de la musique par le cerveau, tout se passe comme si d'un côté 
nous avions le cerveau - humain - des auditeurs de musique, et de l'autre côté, la musique, dont on passe sous silence, sciemment sans doute, son origine, comme si elle était sortie de nulle part, produit sorti casqué de la nature, non présentée, ici en tout cas, comme provenant d'un humain. Sans quoi, étudier les relations entre la construction de la musique par le cerveau - ou la pensée - et le traitement de la réception de la musique par le même cerveau - ou du moins, qui a en partage des universaux cognitifs repérés et énumérés par les sciences cognitives - ne reviendrait-il pas à étudier les universaux cognitifs entre deux cerveaux de nature semblable? La musique, dans un tel travail, serait alors un simple prétexte à la démonstration par l'exemple de l'existence d'universaux cognitifs humains.

Revenons à présent sur cette confusion entre pensée et cerveau; il semble que lorsqu'on parle d'invariants de la pensée - ses limites, sa tendance à classer, à hiérarchiser etc. - on ne parle pas du cerveau, l'organe, mais bien de la pensée.

Peut-être cette confusion pourrait expliquer l'interrogation des sciences cognitives devant le fait que la musique et le langage (faculté à s'exprimer en langue) semblent activer des parties communes du cerveau, alors que l'on peut, après un accident cérébral, souffrir d'amusie sans souffrir de troubles du langage ou inversement. En effet,

De nombreuses études sur les fondements biologiques de la musique ont montré un recouvrement des réseaux neuronaux impliqués dans le traitement de la musique et du langage. Par exemple, certaines études ont appliqué à la musique des méthodes expérimentales utilisées pour le langage, notamment en introduisant un événement qui respecte les structures musicales. Ainsi, par électroencéphalographie - une technique qui mesure l'activité électrique des neurones sur le scalp et qui a une très bonne résolution temporelle -, on a montré que le cerveau réagit rapidement après une violation musicale (en 200 millisecondes après le début du son). Cette réaction est semblable à celle observée après la violation d'une structure syntaxique dans des phrases. (Tillman $2008: 130$ )

Les chercheurs notent cependant un fait bien curieux :

Toutefois, Isabelle Peretz, de l'Université de Montréal, et d'autres chercheurs défendent l'hypothèse que certains réseaux neuronaux sont spécifiques à la musique et séparés de ceux du langage. Notamment, certains patients souffrant de lésions cérébrales - après un accident cérébral vasculaire ou un traumatisme présentent des troubles du langage (nommés aphasie) sans troubles du traitement de la musique. Et d'autres patients ont les symptômes inverses : ils ne rencontrent aucune difficulté avec le langage, mais montrent des troubles de traitement de la musique (nommés amusie). (Tillman $2008: 130$ )

Remarquons comme l'emploi de « inverse » témoigne du parti pris du chercheur : malgré l'étonnement et le constat, les deux symptômes demeurent reliés l'un à l'autre par on ne sait quelle ressemblance ontologique; ils sont opposés comme les deux parties d'un même couple.

Les personnes en souffrant (d'amusie) comprennent normalement la parole et reconnaissent des voix et des sons de l'environnement. En revanche, elles sont incapables de reconnaître une mélodie familière sans paroles ni de détecter une fausse note ou quelqu'un qui chante faux (y compris elles-mêmes). (Tillman 2008 :

Il nous semble que cela s'explique précisément par le fait que ce qui est l'essence de la musique ne correspond justement pas à ce que, de la musique, on peut assimiler aux langues ou au langage. Le cerveau humain - comprenons la pensée - continue de pouvoir organiser des éléments (entre autres, linguistiques) et de comprendre l'organisation 
d'éléments (entre autres, linguistiques), il peut les classer, les hiérarchiser, mais il ne comprend pas la musique. Mais la réception de la musique et sa « compréhension » n'ont peut-être aucun rapport avec ce qui, dans ce qui constitue sa matière, est classable, hiérarchisable et mesurable. Peut-être l'assimilable, le comparable, le mesurable ou le quantifiable en musique correspond-t-il justement à ce qui, dans la musique, est le moins «musical»?

Comparer la musique à une langue ou un langage n'apporte visiblement pas de connaissance pertinente concernant la musique. Nous nous interrogeons sur ce qu'apporte à la connaissance de l'une ou de l'autre discipline ou phénomène, à savoir le langage d'une part, la musique de l'autre, d'être assimilé occasionnellement l'une à l'autre. Loin de mieux définir la musique quand on lui prête certaines fonctions du langage, il semble qu'on lui ôte ce qu'elle a justement de particulier par rapport au langage.

70 Stravinski (1935: 63-64) qualifiait ainsi la musique « impuissante à exprimer quoi que ce soit » institue « un ordre entre l'homme et le temps ».

\section{Conclusion}

71 En réalité, cette conception de la musique, qui en fait un langage, reflète de façon évidente l'idéologie d'une époque, qui aurait traversé quelque deux siècles pour perdurer jusqu'à nos jours. Elle naît au cœur du romantisme et de son développement germanique. La musique répond à cette quête mystique du monde entamée par le romantisme, et en particulier le romantisme allemand. La science déçoit; on découvre les fondements historiques et douteux des écrits religieux, dont on remet en cause l'aspect sacré; les recherches en linguistique, philologie et grammaire comparée conduisent aux hypothèses sur le peuple indo-européen et sa proto-langue. La musique devient le creuset d'une expression mystique du monde, de son essence, de sa vérité.

La musique, pour les romantiques, devient donc un langage au sens fort du terme. L'origine de cette thèse se trouve d'abord dans l'idée selon laquelle tous les langages particuliers de l'humanité ont leur origine dans un langage commun, qui n'était pas encore conceptuel, mais proprement musical - au sens où, comme on le verra avec Nietzsche et Wagner, c'est la sonorité du mot qui renvoie à la chose ou au sentiment qu'elle évoque d'une manière immédiate. (Dufour 2005 : 38)

Il ne s'agit pas de créer des analogies mais de se fonder sur les analogies comme si elles étaient révélatrices d'une essence véritable du monde.

La musique, donc, devient non seulement un langage, mais le seul langage adéquat qui puisse exprimer l'essence du monde. Partant, elle équivaut à un mode de connaissance et elle peut recevoir la qualification de «vraie » ou de «fausse » - on verra que, chez Nietzsche, la musique wagnérienne est qualifiée d'« art vrai ». C'est ce point qu'il nous faut désormais développer.

Que la musique constitue une forme de pensée et un langage n'est pas une thèse propre aux romantiques. Chez les Grecs et pour les théologiens du Moyen-âge, la musique se révèle déjà être une expression du monde tout entier. Mais pour eux, si la musique possède ce pouvoir, ce n'est pas en elle-même et par elle-même, mais du fait qu'elle est réductible à une structure mathématique. Le son, du fait de sa dimension mathématique, n'est rien d'autre qu'un ensemble de rapports qui expriment le cosmos. C'est donc un simple isomorphisme, une correspondance biunivoque entre le cosmos et la musique qui permet de parler de "langage » à propos de la musique. (Dufour 2005 : 31) 

la philosophie ou de la musique, apporte la meilleure connaissance du monde. Nous sommes donc plongés dans une conception très particulière de la musique qui exclut, de fait, certain type de composition ou de création artistique. La musique imitative de Haydn est moquée comme ne révélant rien de l'essence du monde, mais copiant trop simplement sa simple apparence. Nous ne nous plaçons pas dans une recherche mystique de l'essence du monde; néanmoins, nous prétendons apporter quelques lumières sur ce qu'est la musique et ce que sont la langue et le langage. Il est important de souligner comment et à la faveur de quel contexte historique, artistique et intellectuel, les penseurs d'une époque purent rapprocher les domaines et tisser des liens entre les phénomènes pour servir une idéologie, en poursuivant par la re-définition complète du phénomène en question. Il est courant, en effet, d'entendre de nos contemporains proposer d'abord une définition de la musique - simple exemple mais également sujet de nombreuses discordes - pour ensuite en écarter ou y intégrer ce qui illustrera leur définition, tout en faisant comme si les exemples étaient des preuves de la définition qu'ils ont eux-mêmes donnée en début de démonstration. peuvent désigner des objets du réel, peuvent servir une argumentation, peuvent exprimer des idées. S'il était si facile d'exprimer cela en musique, pourquoi tout simplement ne le faisons-nous pas? Pourquoi n'argumente-t-on pas en musique? Pourquoi ne choisissons-nous pas ce «canal»? Pourquoi ne traduisons-nous pas la «musique » en langue pour les personnes frappées d'amusie?

pourra arguer du fait que certaines peuples s'expriment à l'aide de sons qui, à nos oreilles, sonnent de façon harmonieuse ou disharmonieuse, mais en tout cas musicale. Peut-être le fait qu'il s'agisse de nos oreilles n'entre-t-il pas en cause et peut-être les peuples en question entendent également quelque chose de musical. Il n'empêche que si l'expression en langue peut se faire musique, à l'usage, dans certaines de ses fonctions, dans certaines conditions, cela n'implique aucunement que la musique soit un langage ou une langue. Un énoncé en langue peut se doubler d'une musique qui ajoutera quelque chose à l'interprétation de l'ensemble ; cela ne permet pas de dire que la musique est une langue, tout comme cela ne permet pas de dire que la langue est une musique. communicative à la musique pour l'assimiler à un langage ou à une langue révèle peutêtre une idéologie actuelle promouvant l'importance dont la communication et ses outils bénéficient aujourd'hui.

- la nécessaire distinction entre langue et langage, et nous nous appuierons sur ces fondements pour d'ultérieurs travaux en linguistique ;

- la nécessaire distinction entre cerveau et pensée; nous avons mis en garde contre le réflexe qui consiste à penser observer un phénomène du monde alors que l'on n'observe que soi en train d'observer ce phénomène ;

- une proposition d'explication pour l'assimilation de langue ou langage à musique; elle proviendrait d'une mise en valeur des éléments qui sont justement assimilables, mais qui, par là même, sont peut-être ceux des éléments les moins caractéristiques de l'objet à 
définir ;

- l'influence de l'idéologie dans la définition d'un phénomène observé quel qu'il soit.

Cette mise en valeur, par l'analogie, est selon nous le témoin d'une idéologie et d'une volonté d'argumenter en faveur de cette idéologie. Ce que l'on va trouver essentiel, alors, dans la musique, à la faveur de l'analogie présentée ci-dessus, c'est qu'elle est constituée de parties et qu'elle exprime quelque chose.

81 Notre propos n'est pas de montrer en quoi et comment une telle définition de la musique serait erronée ou inexacte, mais d'analyser ce que révèle de l'idéologie du théoricien ou du chercheur une telle définition de la musique.

\section{BIBLIOGRAPHIE}

Chmelik Erzsébet, L'idéologie dans les mots. Contribution à une description topique justifiée par des tests sémantiques, Thèse de doctorat sous la direction de Raccah Pierre-Yves, 2007.

De Schloezer Boris, Introduction à J.-S. Bach ; essai d'esthétique musicale, Rennes, Presses Universitaires de Rennes, 1947.

Dufour Eric, L'esthétique musicale de Nietzsche, Lille, Presses Universitaires du Septentrion, 2005.

Fubini Enrico, « Nature et histoire dans le langage musical », Philosophie, ${ }^{\circ}$ 59, Paris, Minuit, 1998.

Meyer Léonard B., « L'objet musical et l'universel », Philosophie, n 59, Paris, Minuit, 1998.

Platon, Philèbe, texte établi et traduit par Auguste Dies, Paris, les Belles Lettres, Collection Budé, 1978.

Popper Karl, La logique de la découverte scientifique, Paris, Payot, 1973.

Raccah Pierre-Yves, «L'argumentation sans la preuve : Prendre son biais dans la langue », Cognition et Interaction, vol. 2, $\mathrm{n}^{\text {os }} 1-2,1998$, pp. 237-264.

Raccah Pierre-Yves, « Une sémantique du point de vue : de l'intersubjectivité à l'adhésion », in « L'Énonciation identitaire : entre l'individuel et le collectif », dir. par Danielle Forget, Discours social, vol. XXI, 2005, pp. 205-242.

Rastier François, « Communication ou transmission ? » Césure, nº 8, 1995, pp. 151-195.

Riegel Martin, Pellat Jean-Christophe et Rioul René, Grammaire méthodique du français, Paris, PUF Quadrige, 1994.

Ruwet Nicolas, Langage, musique et poésie, Paris, Seuil, 1972.

Stravinski Igor, Chroniques de ma vie, Paris, Denoël, 1935.

Tillman Barbara, « La musique, un langage universel ? », Pour la Science, numéro spécial Sons \&

Musique, de l'art à la science, $\mathrm{n}^{\circ} 373,2008, \mathrm{pp} .124-131$. 


\section{NOTES}

1. Philèbe, texte établi et traduit par Auguste Diès, 1978. Le traducteur croit bon d'ajouter une citation de M. Grammont, Traité de phonétique, 125-126: «il y a pourtant, entre la parole et la musique, deux différences essentielles: "la musique n'emploie que des notes séparées par des intervalles définis; la parole emploie tous les sons compris dans le registre de la voix parlée. Dans la musique, [...] les notes ont toujours une durée appréciable à l'oreille. Dans la parole, les intervalles musicaux sont quelconques et les notes sont souvent très fugitives." ".

2. Ni dans le sens de faculté cognitive, ni dans le sens de système formel.

3. Gardons à l'esprit que Popper emploie « le langage » comme « la faculté humaine d'utiliser les langues ».

4. Il faut comprendre ici que Popper emploie «les langages humains» dans le sens de ce que nous avons défini comme « les langues».

5. Quasi una fantasia, traduction de Jean-Louis Leleu, pp. 4-5, Gallimard, Paris, 1982.

\section{RÉSUMÉS}

Souvent, les langues, les langages et le Langage sont confondus au profit d'une analogie simplifiée qui les associent à la musique. Il est fréquent, en effet, d'entendre, la musique est une langue, ou un langage, ou encore le langage de la musique. Cet article ne vise pas seulement à exposer certaines raisons qui expliqueraient ces analogies abusives, mais également à distinguer plus nettement le langage, les langages et les langues. Au travers de cette étude discriminante, nous présentons et confrontons le cadre théorique de linguistes, de philosophes, de scientifiques de la cognition et de musicologues. La Sémantique des Points de Vue telle qu'elle est développée par Pierre-Yves Raccah fournit le cadre de référence auquel les autres théories sont confrontées. Au-delà de simples différences de définition ou de description, nous avons mis au jour les idéologies révélées par ces mêmes définitions ou descriptions de la musique lorsqu'elle est décrite comme une langue ou langage.

Often the tongues the languages and the language CAN be confused to the benefit of a simplified analogy being associated to music as a tongue. We are often told that music is a tongue or a language anyway or just the language of music. This article not only aims at revealing some reasons to explain false analogies but also at distinguishing better the tongues the languages and the language. Throughout this discriminating study we present and face the theory of linguists, of philosophers, of scientists of cognition and of musicologists. The semantic of point of view presented by Pierre-Yves Raccah gave references to which the other theories are up against. Beyond the simple differences of definition and of description we have brought up to date the ideologies revealed by these same definitions or descriptions of music where it is described as a tongue or a language. 
INDEX

Mots-clés : langue, langage, musique, théorie, description, définition

Keywords : tongue, language, music, theory

\section{AUTEUR}

LAETITIA PILLE

Université d'Orléans, LLL 